PDES, SUBMANIFOLDS AND

AFFINE DIFFERENTIAL GEOMETRY

BANACH CENTER PUBLICATIONS, VOLUME 69

INSTITUTE OF MATHEMATICS

POLISH ACADEMY OF SCIENCES

WARSZAWA 2005

\title{
ON THE MEASURABILITY \\ OF SETS OF PAIRS OF STRAIGHT LINES IN THE SIMPLY ISOTROPIC SPACE
}

\author{
MARGARITA G. SPIROVA \\ Dept. of Descriptive Geometry, Univ. of Architecture, Civil Eng. and Geodesy \\ 1, Christo Smirnenski Blvd., 1046 Sofia, Bulgaria \\ E-mail: spirov_fgs@uacg.bg \\ ADRIJAN V. BORISOV \\ Dept. of Mathematics, South-West University "Neofit Rilski" \\ 66, Ivan Mihailov St., 2700 Blagoevgrad, Bulgaria \\ E-mail: adribor@aix.swu.bg
}

\begin{abstract}
We study the measurability of sets of pairs of straight lines with respect to the group of motions in the simply isotropic space $I_{3}^{(1)}$ by solving PDEs. Also some Crofton type formulas are obtained for the corresponding densities.
\end{abstract}

1. Introduction. The simply isotropic space $I_{3}^{(1)}$ is defined (see [7]) as a projective space $P_{3}(R)$ in which the absolute consists of a plane $\omega$ (the absolute plane) and two complex conjugate straight lines $f_{1}, f_{2}$ (the absolute lines) in $\omega$ with a real intersection point $F$ (the absolute point). In homogeneous coordinates $\left(x_{0}, x_{1}, x_{2}, x_{3}\right)$ we can take the plane $x_{0}=0$ as the plane $\omega$, the line $x_{0}=0, x_{1}+i x_{2}=0$ as the line $f_{1}$, the line $x_{0}=0, x_{1}-i x_{2}=0$ as the line $f_{2}$ and the point $(0,0,0,1)$ as the point $F$. The 6 -parameter group $B_{6}^{(1)}$ of transformations (in affine coordinates $(x, y, z)$ )

$$
\begin{aligned}
& x^{\prime}=c_{1}+x \cos \varphi-y \sin \varphi, \\
& y^{\prime}=c_{2}+x \sin \varphi+y \cos \varphi, \\
& z^{\prime}=c_{3}+c_{4} x+c_{5} y+z,
\end{aligned}
$$

where $c_{1}, c_{2}, c_{3}, c_{4}, c_{5}, \varphi \in R$, is called the group of simply isotropic motions in $I_{3}^{(1)}$.

2000 Mathematics Subject Classification: Primary 53C65.

Key words and phrases: measurability, density, simply isotropic space.

The paper is in final form and no version of it will be published elsewhere. 
We emphasize that much of the material which is common in the geometry of the simply isotropic space $I_{3}^{(1)}$ can be found in [7]. Using some basic concepts of integral geometry in the sense of M. I. Stoka [9], [10], G. I. Drinfel'd and A. V. Lucenko [4], [5], [6], we study the measurability of sets of pairs of straight lines in $I_{1}^{(3)}$. Analogous problems for points and planes in $I_{3}^{(1)}$ have been treated in [2].

2. Measurability of a set of pairs of skew straight lines. A straight line is said to be (completely) isotropic if its infinite point coincides with the absolute point $F$; otherwise the straight line is said to be nonisotropic $[7 ;$ p. 5$]$.

Let $G_{1}$ and $G_{2}$ be two nonisotropic straight lines and denote by $U_{1}$ and $U_{2}$ their infinite points, respectively. Then $G_{1}$ and $G_{2}$ are said to be of type $\alpha$ or of type $\beta$ if the points $U_{1}, U_{2}$ and $F$ are noncollinear or collinear, respectively [7; p. 45].

2.1. Density of pairs of skew nonisotropic straight lines of type $\alpha$. Let $\left(G_{1}, G_{2}\right)$ be a pair of skew nonisotropic straight lines of type $\alpha$ determined by the equations

$$
G_{i}: \quad x=a_{i} z+p_{i}, \quad y=b_{i} z+q_{i}, \quad i=1,2,
$$

where

$$
\begin{gathered}
\left(a_{2}-a_{1}\right)\left(q_{2}-q_{1}\right)-\left(b_{2}-b_{1}\right)\left(p_{2}-p_{1}\right) \neq 0, \\
a_{1} b_{2}-a_{2} b_{1} \neq 0 .
\end{gathered}
$$

Under the action of $(1)$ the pair $\left(G_{1}, G_{2}\right)\left(a_{1}, b_{1}, p_{1}, q_{1}, a_{2}, b_{2}, p_{2}, q_{2}\right)$ is transformed into the pair $\left(G_{1}^{\prime}, G_{2}^{\prime}\right)\left(a_{1}^{\prime}, b_{1}^{\prime}, p_{1}^{\prime}, q_{1}^{\prime}, a_{2}^{\prime}, b_{2}^{\prime}, p_{2}^{\prime}, q_{2}^{\prime}\right)$ as

$$
\begin{aligned}
& a_{i}^{\prime}=K_{i}\left(a_{i} \cos \varphi-b_{i} \sin \varphi\right), \\
& b_{i}^{\prime}=K_{i}\left(a_{i} \sin \varphi+b_{i} \cos \varphi\right), \\
& p_{i}^{\prime}=K_{i}\left\{\left[-c_{3} a_{i}+p_{i}+c_{5}\left(b_{i} p_{i}-a_{i} q_{i}\right)\right] \cos \varphi+\left[c_{3} b_{i}-q_{i}+c_{4}\left(b_{i} p_{i}-a_{i} q_{i}\right)\right] \sin \varphi\right\}+c_{1}, \\
& q_{i}^{\prime}=K_{i}\left\{\left[-c_{3} a_{i}+p_{i}+c_{5}\left(b_{i} p_{i}-a_{i} q_{i}\right)\right] \sin \varphi-\left[c_{3} b_{i}-q_{i}+c_{4}\left(b_{i} p_{i}-a_{i} q_{i}\right)\right] \cos \varphi\right\}+c_{2},
\end{aligned}
$$

where $K_{i}=\left(1+a_{i} c_{4}+b_{i} c_{5}\right)^{-1}, i=1,2$.

The transformations (3) form the associated group $\bar{B}_{6}^{(1)}$ of $B_{6}^{(1)}[9 ;$ p. 34], [10; p. 17]. $\bar{B}_{6}^{(1)}$ is isomorphic to $B_{6}^{(1)}$ and the invariant density with respect to $B_{6}^{(1)}$ of the pairs of lines $\left(G_{1}, G_{2}\right)$, if it exists, coincides with the invariant density with respect to $\bar{B}_{6}^{(1)}$ of the points $\left(a_{1}, b_{1}, p_{1}, q_{1}, a_{2}, b_{2}, p_{2}, q_{2}\right)$ in the set of parameters. The associated group $\bar{B}_{6}^{(1)}$ has the infinitesimal operators

$$
\begin{aligned}
Y_{1}= & \frac{\partial}{\partial p_{1}}+\frac{\partial}{\partial p_{2}}, \quad Y_{2}=\frac{\partial}{\partial q_{1}}+\frac{\partial}{\partial q_{2}}, \quad Y_{3}=a_{1} \frac{\partial}{\partial p_{1}}+b_{1} \frac{\partial}{\partial q_{1}}+a_{2} \frac{\partial}{\partial p_{2}}+b_{2} \frac{\partial}{\partial q_{2}}, \\
Y_{4}= & b_{1} \frac{\partial}{\partial a_{1}}-a_{1} \frac{\partial}{\partial b_{1}}+q_{1} \frac{\partial}{\partial p_{1}}-p_{1} \frac{\partial}{\partial q_{1}}+b_{2} \frac{\partial}{\partial a_{2}}-a_{2} \frac{\partial}{\partial b_{2}}+q_{2} \frac{\partial}{\partial p_{2}}-p_{2} \frac{\partial}{\partial q_{2}} \\
Y_{5}= & a_{1}^{2} \frac{\partial}{\partial a_{1}}+a_{1} b_{1} \frac{\partial}{\partial b_{1}}+a_{1} p_{1} \frac{\partial}{\partial p_{1}}+b_{1} p_{1} \frac{\partial}{\partial q_{1}}+a_{2}^{2} \frac{\partial}{\partial a_{2}}+a_{2} b_{2} \frac{\partial}{\partial b_{2}} \\
& +a_{2} p_{2} \frac{\partial}{\partial p_{2}}+b_{2} p_{2} \frac{\partial}{\partial q_{2}}, \quad Y_{6}=a_{1} b_{1} \frac{\partial}{\partial a_{1}}+b_{1}^{2} \frac{\partial}{\partial b_{1}}+a_{1} q_{1} \frac{\partial}{\partial p_{1}}+b_{1} q_{1} \frac{\partial}{\partial q_{1}} \\
& +a_{2} b_{2} \frac{\partial}{\partial a_{2}}+b_{2}^{2} \frac{\partial}{\partial b_{2}}+a_{2} q_{2} \frac{\partial}{\partial p_{2}}+b_{2} q_{2} \frac{\partial}{\partial q_{2}} .
\end{aligned}
$$


The associated group $\bar{B}_{6}^{(1)}$ acts intransitively on the set of pairs $\left(G_{1}, G_{2}\right)$ and therefore the pairs $\left(G_{1}, G_{2}\right)$ do not have invariant density under $B_{6}^{(1)}$. The system $Y_{i}(f)=0$, $i=1, \ldots, 6$ has two independent integrals

$$
f^{\prime}=\frac{a_{1} b_{2}-a_{2} b_{1}}{\sqrt{a_{1}^{2}+b_{1}^{2}} \sqrt{a_{2}^{2}+b_{2}^{2}}}, \quad f^{\prime \prime}=\frac{\left(a_{2}-a_{1}\right)\left(q_{2}-q_{1}\right)-\left(b_{2}-b_{1}\right)\left(p_{2}-p_{1}\right)}{a_{1} b_{2}-a_{2} b_{1}}
$$

and (5) are absolute invariants of $\bar{B}_{6}^{(1)}$. It follows that we can define the density for the set of the pairs $\left(G_{1}, G_{2}\right)\left(a_{1}, b_{1}, p_{1}, q_{1}, a_{2}, b_{2}, p_{2}, q_{2}\right)$ of skew nonisotropic straight lines of type $\alpha$ by the equality

$$
\begin{aligned}
d\left(G_{1}, G_{2}\right)= & \left|\frac{\left(a_{2}-a_{1}\right)\left(q_{2}-q_{1}\right)-\left(b_{2}-b_{1}\right)\left(p_{2}-p_{1}\right)}{\sqrt{a_{1}^{2}+b_{1}^{2}} \sqrt{a_{2}^{2}+b_{2}^{2}}}\right| \\
& \times d a_{1} \wedge d b_{1} \wedge d p_{1} \wedge d q_{1} \wedge d a_{2} \wedge d b_{2} \wedge d p_{2} \wedge d q_{2} .
\end{aligned}
$$

REMARK 1. We note that [7; p. 45]

$$
\sin \psi=\frac{a_{1} b_{2}-a_{2} b_{1}}{\sqrt{a_{1}^{2}+b_{1}^{2}} \sqrt{a_{2}^{2}+b_{2}^{2}}}, \quad \delta\left(G_{1}, G_{2}\right)=\frac{\left(a_{2}-a_{1}\right)\left(q_{2}-q_{1}\right)-\left(b_{2}-b_{1}\right)\left(p_{2}-p_{1}\right)}{a_{1} b_{2}-a_{2} b_{1}},
$$

where $\psi$ and $\delta\left(G_{1}, G_{2}\right)$ are the angle and the distance from $G_{1}$ to $G_{2}$, respectively.

Denote $\delta\left(G_{1}, G_{2}\right)=\delta$ and replacing (7) into (6) we find another expression for density:

$$
d\left(G_{1}, G_{2}\right)=|\delta \sin \psi| d a_{1} \wedge d b_{1} \wedge d p_{1} \wedge d q_{1} \wedge d a_{2} \wedge d b_{2} \wedge d p_{2} \wedge d q_{2} .
$$

On the other hand, the set of nonisotropic straight lines $G_{i}\left(a_{i}, b_{i}, p_{i}, q_{i}\right)$ is measurable with respect to the group $B_{6}^{(1)}$ and has the invariant density [2]

$$
d G_{i}=\frac{d a_{i} \wedge d b_{i} \wedge d p_{i} \wedge d q_{i}}{\left(a_{i}^{2}+b_{i}^{2}\right)^{2}}
$$

Then putting (9) in (8) we obtain

$$
d\left(G_{1}, G_{2}\right)=|\delta \sin \psi|\left(a_{1}^{2}+b_{1}^{2}\right)\left(a_{2}^{2}+b_{2}^{2}\right) d G_{1} \wedge d G_{2} .
$$

Assume that the straight line $G_{i}$ has the angle $\varphi_{i}$ with the horizontal plane $O x y$ and $\widetilde{G_{i}}$ denotes the orthogonal projection of $G_{i}$ on $O x y$. Then $[7 ;$ p. 48]

$$
\varphi_{i}=\frac{1}{\sqrt{a_{i}^{2}+b_{i}^{2}}}, \quad \widetilde{G_{i}}: \quad b_{i} x-a_{i} y+a_{i} q_{i}-b_{i} p_{i}=0, \quad z=0
$$

and $[2]$

$$
d G_{i}=\frac{1}{\left|a_{i}\right|} d \widetilde{G_{i}} \wedge d \varphi_{i} \wedge d p_{i}=\frac{1}{\left|b_{i}\right|} d \widetilde{G_{i}} \wedge d \varphi_{i} \wedge d q_{i}
$$

where

$$
d \widetilde{G_{i}}=\frac{1}{\left(a_{i}^{2}+b_{i}^{2}\right)^{\frac{3}{2}}}\left(b_{i}^{2} d a_{i} \wedge d p_{i}-a_{i} b_{i} d a_{i} \wedge d q_{i}-a_{i} b_{i} d b_{i} \wedge d p_{i}+a_{i}^{2} d b_{i} \wedge d q_{i}\right)
$$

is the density for $\widetilde{G_{i}}$ in $O x y$. Note that the plane $O x y$ is Euclidean and $d \widetilde{G_{i}}$ is the metric density for the straight lines in $O x y$ [8; p. 29], [9; p. 66].

Applying (11) and (12) to (10), we get

$$
d\left(G_{1}, G_{2}\right)=\left|\frac{\delta \sin \psi}{\varphi_{1}^{4} \varphi_{2}^{4}}\right| d G_{1} \wedge d G_{2}
$$


and

$$
\begin{aligned}
d\left(G_{1}, G_{2}\right) & =\left|\frac{\delta \sin \psi}{a_{1} a_{2} \varphi_{1}^{4} \varphi_{2}^{4}}\right| d \widetilde{G_{1}} \wedge d \widetilde{G_{2}} \wedge d \varphi_{1} \wedge d \varphi_{2} \wedge d p_{1} \wedge d p_{2} \\
& =\left|\frac{\delta \sin \psi}{a_{1} b_{2} \varphi_{1}^{4} \varphi_{2}^{4}}\right| d \widetilde{G_{1}} \wedge d \widetilde{G_{2}} \wedge d \varphi_{1} \wedge d \varphi_{2} \wedge d p_{1} \wedge d q_{2} \\
& =\left|\frac{\delta \sin \psi}{b_{1} a_{2} \varphi_{1}^{4} \varphi_{2}^{4}}\right| d \widetilde{G_{1}} \wedge d \widetilde{G_{2}} \wedge d \varphi_{1} \wedge d \varphi_{2} \wedge d q_{1} \wedge d p_{2} \\
& =\left|\frac{\delta \sin \psi}{b_{1} b_{2} \varphi_{1}^{4} \varphi_{2}^{4}}\right| d \widetilde{G_{1}} \wedge d \widetilde{G_{2}} \wedge d \varphi_{1} \wedge d \varphi_{2} \wedge d q_{1} \wedge d q_{2} .
\end{aligned}
$$

If we denote $\overline{P_{1}}=G_{1} \cap O x y, \overline{P_{2}}=G_{2} \cap O x y$, then on the plane $O x y$ we have

$$
d \bar{P}_{1}=d p_{1} \wedge d q_{1}, \quad d \bar{P}_{2}=d p_{2} \wedge d q_{2} .
$$

By differentiation of (7) and (11) and by exterior multiplication of (15) we get

$$
d \delta \wedge d \psi \wedge d \varphi_{1} \wedge d \varphi_{2} \wedge d \bar{P}_{1} \wedge d \bar{P}_{2}
$$

$=\frac{\sin \psi\left[\left(a_{2}-a_{1}\right)\left(p_{2}-p_{1}\right)+\left(b_{2}-b_{1}\right)\left(q_{2}-q_{1}\right)\right]}{\varphi_{1}^{2} \varphi_{2}^{2}} d a_{1} \wedge d b_{1} \wedge d p_{1} \wedge d q_{1} \wedge d a_{2} \wedge d b_{2} \wedge d p_{2} \wedge d q_{2}$.

Inserting (16) into (8) we obtain

$$
\begin{aligned}
& d\left(G_{1}, G_{2}\right) \\
& \quad=\left|\frac{\delta \varphi_{1}^{2} \varphi_{2}^{2}}{\left(a_{2}-a_{1}\right)\left(p_{2}-p_{1}\right)+\left(b_{2}-b_{1}\right)\left(q_{2}-q_{1}\right)}\right| d \delta \wedge d \psi \wedge d \varphi_{1} \wedge d \varphi_{2} \wedge d \bar{P}_{1} \wedge d \bar{P}_{2} .
\end{aligned}
$$

We summarize the foregoing results in the following

TheOREM 1. The density for the pairs $\left(G_{1}, G_{2}\right)$ of skew nonisotropic straight lines of

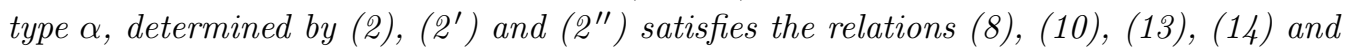
(17).

2.2. Density of pairs of skew nonisotropic straight lines of type $\beta$. Let $\left(G_{1}, G_{2}\right)$ be a pair of skew nonisotropic straight lines of type $\beta$ determined by $(2),\left(2^{\prime}\right)$ and the equality

$$
a_{1} b_{2}-a_{2} b_{1}=0
$$

Without loss of generality we can assume that $a_{1} \neq 0$ and then we have

$$
b_{2}=\frac{a_{2}}{a_{1}} b_{1} \text {. }
$$

From (2) and (18) it follows that the pairs $\left(G_{1}, G_{2}\right)$ are determined by the equations

$$
\begin{array}{ll}
G_{1}: & x=a_{1} z+p_{1}, \quad y=b_{1} z+q_{1}, \quad a_{1} \neq 0, \\
G_{2}: & x=a_{2} z+p_{2}, \quad y=\frac{a_{2}}{a_{1}} b_{1} z+q_{2}, \quad a_{2} \neq 0 .
\end{array}
$$

Now, under the action of $(1)$, the pair $\left(G_{1}, G_{2}\right)\left(a_{1}, b_{1}, p_{1}, q_{1}, a_{2}, p_{2}, q_{2}\right)$ is transformed into the pair $\left(G_{1}^{\prime}, G_{2}^{\prime}\right)\left(a_{1}^{\prime}, b_{1}^{\prime}, p_{1}^{\prime}, q_{1}^{\prime}, a_{2}^{\prime}, p_{2}^{\prime}, q_{2}^{\prime}\right)$ and the corresponding associated group $\bar{B}_{6}^{(1)}$ 
has the infinitesimal operators (see (4))

$$
\begin{aligned}
& Z_{1}=\frac{\partial}{\partial p_{1}}+\frac{\partial}{\partial p_{2}}, \quad Z_{2}=\frac{\partial}{\partial q_{1}}+\frac{\partial}{\partial q_{2}}, \quad Z_{3}=a_{1} \frac{\partial}{\partial p_{1}}+b_{1} \frac{\partial}{\partial q_{1}}+a_{2} \frac{\partial}{\partial p_{2}}+\frac{a_{2}}{a_{1}} b_{1} \frac{\partial}{\partial q_{2}} \\
& Z_{4}=b_{1} \frac{\partial}{\partial a_{1}}-a_{1} \frac{\partial}{\partial b_{1}}+q_{1} \frac{\partial}{\partial p_{1}}-p_{1} \frac{\partial}{\partial q_{1}}+\frac{a_{2}}{a_{1}} b_{1} \frac{\partial}{\partial a_{2}}+q_{2} \frac{\partial}{\partial p_{2}}-p_{2} \frac{\partial}{\partial q_{2}} \\
& Z_{5}=a_{1}^{2} \frac{\partial}{\partial a_{1}}+a_{1} b_{1} \frac{\partial}{\partial b_{1}}+a_{1} p_{1} \frac{\partial}{\partial p_{1}}+b_{1} p_{1} \frac{\partial}{\partial q_{1}}+a_{2}^{2} \frac{\partial}{\partial a_{2}}+a_{2} p_{2} \frac{\partial}{\partial p_{2}}+\frac{a_{2}}{a_{1}} b_{1} p_{2} \frac{\partial}{\partial q_{2}} \\
& Z_{6}=a_{1} b_{1} \frac{\partial}{\partial a_{1}}+b_{1}^{2} \frac{\partial}{\partial b_{1}}+a_{1} q_{1} \frac{\partial}{\partial p_{1}}+b_{1} q_{1} \frac{\partial}{\partial q_{1}}+\frac{a_{2}^{2}}{a_{1}} b_{1} \frac{\partial}{\partial a_{2}}+a_{2} q_{2} \frac{\partial}{\partial p_{2}}+\frac{a_{2}}{a_{1}} b_{1} q_{2} \frac{\partial}{\partial q_{2}}
\end{aligned}
$$

Since $\bar{B}_{6}^{(1)}$ acts intransitively on the set of pairs $\left(G_{1}, G_{2}\right)$ it follows that the pairs $\left(G_{1}, G_{2}\right)$ do not have invariant density with respect to $B_{6}^{(1)}$. The system $Z_{i}(f)=0, i=1, \ldots, 6$ has two independent integrals

$$
f^{\prime}=\frac{b_{1}\left(p_{2}-p_{1}\right)-a_{1}\left(q_{2}-q_{1}\right)}{\sqrt{a_{1}^{2}+b_{1}^{2}}}, \quad f^{\prime \prime}=\frac{a_{1}-a_{2}}{a_{2} \sqrt{a_{1}^{2}+b_{1}^{2}}}
$$

that are absolute invariants of $\bar{B}_{6}^{(1)}$.

Now we can define the density for the pairs $\left(G_{1}, G_{2}\right)\left(a_{1}, b_{1}, p_{1}, q_{1}, a_{2}, p_{2}, q_{2}\right)$ of skew nonisotropic straight lines of type $\beta$ by the equality $d\left(G_{1}, G_{2}\right)=\left|\frac{\left(a_{1}-a_{2}\right)\left[b_{1}\left(p_{2}-p_{1}\right)-a_{1}\left(q_{2}-q_{1}\right)\right]}{a_{2}\left(a_{1}^{2}+b_{1}^{2}\right)}\right| d a_{1} \wedge d b_{1} \wedge d p_{1} \wedge d q_{1} \wedge d a_{2} \wedge d p_{2} \wedge d q_{2}$.

REMARK 2. We note that $[7 ;$ p. 45-46]

$$
a=\frac{b_{1}\left(p_{2}-p_{1}\right)-a_{1}\left(q_{2}-q_{1}\right)}{\sqrt{a_{1}^{2}+b_{1}^{2}}}, \quad s=\frac{a_{1}-a_{2}}{a_{2} \sqrt{a_{1}^{2}+b_{1}^{2}}}
$$

are the distance and the angle from $G_{1}$ to $G_{2}$, respectively. It follows that

$$
d\left(G_{1}, G_{2}\right)=|a s| d a_{1} \wedge d b_{1} \wedge d p_{1} \wedge d q_{1} \wedge d a_{2} \wedge d p_{2} \wedge d q_{2}
$$

By differentiation of (11) and (20) and by exterior multiplication of (15) we find

$$
\begin{aligned}
d a \wedge d s & \wedge d \varphi_{1} \wedge d \bar{P}_{1} \wedge d \bar{P}_{2} \\
& =\left|\frac{s^{2} a_{1}\left[a_{1}\left(p_{2}-p_{1}\right)+b_{1}\left(q_{2}-q_{1}\right)\right]}{\left(a_{1}-a_{2}\right)^{2} \varphi_{1}^{3}}\right| d a_{1} \wedge d b_{1} \wedge d p_{1} \wedge d q_{1} \wedge d a_{2} \wedge d p_{2} \wedge d q_{2} .
\end{aligned}
$$

Putting (22) into (21) we obtain

$$
d\left(G_{1}, G_{2}\right)=\left|\frac{a\left(a_{1}-a_{2}\right)^{2} \varphi_{1}^{3}}{s a_{1}\left[a_{1}\left(p_{2}-p_{1}\right)+b_{1}\left(q_{2}-q_{1}\right)\right]}\right| d a \wedge d s \wedge d \varphi_{1} \wedge d \bar{P}_{1} \wedge d \bar{P}_{2} .
$$

So we can state:

TheOREM 2. The density for the pairs $\left(G_{1}, G_{2}\right)$ of skew nonisotropic straight lines of type $\beta$, determined by (19), satisfies the relations (21) and (23).

2.3. Density of pairs of skew nonisotropic and isotropic straight lines. Let $\left(G_{1}, G_{2}\right)$ be a pair of the skew nonisotropic straight line $G_{1}: x=a_{1} z+p_{1}, y=b_{1} z+q_{1}$ and the isotropic straight line $G_{2}: x=p_{2}, y=q_{2}$, where $b_{1}\left(p_{2}-p_{1}\right)-a_{1}\left(q_{2}-q_{1}\right) \neq 0$. The 
corresponding associated group $\bar{B}_{6}^{(1)}$ has the infinitesimal operators

$$
\begin{aligned}
U_{1} & =\frac{\partial}{\partial p_{1}}+\frac{\partial}{\partial p_{2}}, \quad U_{2}=\frac{\partial}{\partial q_{1}}+\frac{\partial}{\partial q_{2}}, \quad U_{3}=a_{1} \frac{\partial}{\partial p_{1}}+b_{1} \frac{\partial}{\partial q_{1}}, \\
U_{4} & =b_{1} \frac{\partial}{\partial a_{1}}-a_{1} \frac{\partial}{\partial b_{1}}+q_{1} \frac{\partial}{\partial p_{1}}-p_{1} \frac{\partial}{\partial q_{1}}+q_{2} \frac{\partial}{\partial p_{2}}-p_{2} \frac{\partial}{\partial q_{2}}, \\
U_{5} & =a_{1}^{2} \frac{\partial}{\partial a_{1}}+a_{1} b_{1} \frac{\partial}{\partial b_{1}}+a_{1} p_{1} \frac{\partial}{\partial p_{1}}+b_{1} p_{1} \frac{\partial}{\partial q_{1}}, \\
U_{6} & =a_{1} b_{1} \frac{\partial}{\partial a_{1}}+b_{1}^{2} \frac{\partial}{\partial b_{1}}+a_{1} q_{1} \frac{\partial}{\partial p_{1}}+b_{1} q_{1} \frac{\partial}{\partial q_{1}} .
\end{aligned}
$$

It is easy to verify that $\bar{B}_{6}^{(1)}$ acts intransitively on the set of pairs $\left(G_{1}, G_{2}\right)$ and therefore the pairs $\left(G_{1}, G_{2}\right)$ do not have invariant density under $B_{6}^{(1)}$. The system $U_{i}(f)=0, i=$ $=1, \ldots, 6$ has the solution

$$
f=\frac{b_{1}\left(p_{2}-p_{1}\right)-a_{1}\left(q_{2}-q_{1}\right)}{\sqrt{a_{1}^{2}+b_{1}^{2}}}
$$

that is an absolute invariant of $\bar{B}_{6}^{(1)}$.

We define the density of the pairs $\left(G_{1}, G_{2}\right)\left(a_{1}, b_{1}, p_{1}, q_{1}, p_{2}, q_{2}\right)$ by the equality

$$
d\left(G_{1}, G_{2}\right)=\left|\frac{b_{1}\left(p_{2}-p_{1}\right)-a_{1}\left(q_{2}-q_{1}\right)}{\sqrt{a_{1}^{2}+b_{1}^{2}}}\right| d a_{1} \wedge d b_{1} \wedge d p_{1} \wedge d q_{1} \wedge d p_{2} \wedge d q_{2} .
$$

REMARK 3. We note that $[7 ;$ p. 46]

$$
l=\frac{b_{1}\left(p_{2}-p_{1}\right)-a_{1}\left(q_{2}-q_{1}\right)}{\sqrt{a_{1}^{2}+b_{1}^{2}}}
$$

is the distance from $G_{1}$ to $G_{2}$ and consequently (24) can be written in the form

$$
d\left(G_{1}, G_{2}\right)=|l| d a_{1} \wedge d b_{1} \wedge d p_{1} \wedge d q_{1} \wedge d p_{2} \wedge d q_{2} .
$$

On the other hand, differentiating (11) (for $i=1$ ) and (25) and by exterior multiplication of (15) we obtain

(27) $d l \wedge d \varphi_{1} \wedge d \bar{P}_{1} \wedge d \bar{P}_{2}=\frac{a_{1}\left(p_{2}-p_{1}\right)+b_{1}\left(q_{2}-q_{1}\right)}{\left(a_{1}^{2}+b_{1}^{2}\right)^{2}} d a_{1} \wedge d b_{1} \wedge d p_{1} \wedge d q_{1} \wedge d p_{2} \wedge d q_{2}$.

Substituting (27) into (26), we find

$$
d\left(G_{1}, G_{2}\right)=\left|\frac{l \varphi_{1}^{4}}{a_{1}\left(p_{2}-p_{1}\right)+b_{1}\left(q_{2}-q_{1}\right)}\right| d l \wedge d \varphi_{1} \wedge d \bar{P}_{1} \wedge d \bar{P}_{2} .
$$

Thus the following theorem is true:

Theorem 3. The density for the pairs $\left(G_{1}, G_{2}\right)$ of the skew nonisotropic straight line $G_{1}: x=a_{1} z+p_{1}, y=b_{1} z+q_{1}$ and the isotropic straight line $G_{2}: x=p_{2}, y=q_{2}$ satisfies the relations (26) and (28).

\section{Measurability of a set of pairs of intersecting straight lines}

3.1. Density of pairs of intersecting nonisotropic straight lines of type $\alpha$. Let $\left(G_{1}, G_{2}\right)$ be a pair of intersecting nonisotropic straight lines of type $\alpha$ given by 


$$
\begin{array}{ll}
G_{1}: & x=a_{1} z+p-a_{1} r, \quad y=b_{1} z+q-b_{1} r, \\
G_{2}: & x=a_{2} z+p-a_{2} r, \quad y=b_{2} z+q-b_{2} r,
\end{array}
$$

i.e. $G_{1} \cap G_{2}=P(p, q, r)$ and $\left(2^{\prime \prime}\right)$ is true. The corresponding associated group $\bar{B}_{6}^{(1)}$ consists of the transformations

$$
\begin{aligned}
& a_{i}^{\prime}=\left(1+a_{i} c_{4}+b_{i} c_{5}\right)^{-1}\left(a_{i} \cos \varphi-b_{i} \sin \varphi\right), \\
& b_{i}^{\prime}=\left(1+a_{i} c_{4}+b_{i} c_{5}\right)^{-1}\left(a_{i} \sin \varphi+b_{i} \cos \varphi\right), \quad i=1,2, \\
& p^{\prime}=c_{1}+p \cos \varphi-q \sin \varphi \\
& q^{\prime}=c_{2}+p \sin \varphi+q \cos \varphi \\
& r^{\prime}=c_{3}+c_{4} p+c_{5} q+r
\end{aligned}
$$

and has the infinitesimal operators

$$
\begin{aligned}
& Y_{1}=\frac{\partial}{\partial p}, \quad Y_{2}=\frac{\partial}{\partial q}, \quad Y_{3}=\frac{\partial}{\partial r}, \quad Y_{4}=b_{1} \frac{\partial}{\partial a_{1}}-a_{1} \frac{\partial}{\partial b_{1}}+b_{2} \frac{\partial}{\partial a_{2}}-a_{2} \frac{\partial}{\partial b_{2}}+q \frac{\partial}{\partial p}-p \frac{\partial}{\partial q}, \\
& Y_{5}=a_{1}^{2} \frac{\partial}{\partial a_{1}}+a_{1} b_{1} \frac{\partial}{\partial b_{1}}+a_{2}^{2} \frac{\partial}{\partial a_{2}}+a_{2} b_{2} \frac{\partial}{\partial b_{2}}-p \frac{\partial}{\partial r}, \\
& Y_{6}=a_{1} b_{1} \frac{\partial}{\partial a_{1}}+b_{1}^{2} \frac{\partial}{\partial b_{1}}+a_{2} b_{2} \frac{\partial}{\partial a_{2}}+b_{2}^{2} \frac{\partial}{\partial b_{2}}-q \frac{\partial}{\partial r} .
\end{aligned}
$$

The group $\bar{B}_{6}^{(1)}$ is intransitive and therefore the set of pairs of intersecting straight lines (29) is not measurable with respect to $B_{6}^{(1)}$. But the value

$$
f=\frac{a_{1} b_{2}-a_{2} b_{1}}{\sqrt{a_{1}^{2}+b_{1}^{2}} \sqrt{a_{2}^{2}+b_{2}^{2}}}
$$

is an absolute invariant of $\bar{B}_{6}^{(1)}$ and so we can define the density for the pairs $\left(G_{1}, G_{2}\right)$ $\left(a_{1}, b_{1}, a_{2}, b_{2}, p, q, r\right)$ by the equality

$$
d\left(G_{1}, G_{2}\right)=\left|\frac{a_{1} b_{2}-a_{2} b_{1}}{\sqrt{a_{1}^{2}+b_{1}^{2}} \sqrt{a_{2}^{2}+b_{2}^{2}}}\right| d a_{1} \wedge d b_{1} \wedge d a_{2} \wedge d b_{2} \wedge d p \wedge d q \wedge d r .
$$

Replacing (7) to (30) we obtain

$$
d\left(G_{1}, G_{2}\right)=|\sin \psi| d a_{1} \wedge d b_{1} \wedge d a_{2} \wedge d b_{2} \wedge d p \wedge d q \wedge d r .
$$

Denote by $\left(x_{1}, y_{1}, 0\right)$ and $\left(x_{2}, y_{2}, 0\right)$ the coordinates of the points $\bar{P}_{1}=G_{1} \cap O x y$ and $\bar{P}_{2}=G_{2} \cap O x y$, respectively. Since

$$
x_{i}=p-a_{i} r, \quad y_{i}=q-b_{i} r, \quad i=1,2,
$$

then

$$
\begin{aligned}
d \bar{P}_{1} \wedge d \bar{P}_{2} \wedge d P & =d x_{1} \wedge d y_{1} \wedge d x_{2} \wedge d y_{2} \wedge d p \wedge d q \wedge d r \\
& =r^{4} d a_{1} \wedge d b_{1} \wedge d a_{2} \wedge d b_{2} \wedge d p \wedge d q \wedge d r
\end{aligned}
$$

and from (31) and (32) it follows that

$$
d\left(G_{1}, G_{2}\right)=\left|\frac{\sin \psi}{r^{4}}\right| d \bar{P}_{1} \wedge d \bar{P}_{2} \wedge d P .
$$


On the other hand, on the plane $O x y$ we have the classical Blaschke formula [1], [8; p. 59]

$$
d \bar{P}_{1} \wedge d \bar{P}_{2} \wedge d \widetilde{P}=\widetilde{D}^{3} d \widetilde{G}_{1} \wedge d \widetilde{G}_{2} \wedge d \bar{G},
$$

where $\widetilde{P}$ is the orthogonal projection of the point $P$ on the coordinate plane $O x y, \widetilde{G}_{1}=$ $\bar{P}_{1} \widetilde{P}, \widetilde{G}_{2}=\bar{P}_{2} \widetilde{P}, \bar{G}=\bar{P}_{1} \bar{P}_{2}$ and $\widetilde{D}$ is the diameter of the circumscribed circle of the triangle $\bar{P}_{1} \bar{P}_{2} \widetilde{P}$. Since $d P=d \widetilde{P} \wedge d r$, by (33) and (34) we get

$$
d\left(G_{1}, G_{2}\right)=\left|\frac{\widetilde{D}^{3} \sin \psi}{r^{4}}\right| d \widetilde{G}_{1} \wedge d \widetilde{G}_{2} \wedge d \bar{G} \wedge d r .
$$

Further, we have

$$
\widetilde{G}_{i}: \quad b_{i} x-a_{i} y+a_{i} q-b_{i} p=0, \quad z=0
$$

and according to $\left(12^{\prime}\right)$ we can write

$$
d \widetilde{G}_{i}=\frac{1}{\left(a_{i}^{2}+b_{i}^{2}\right)^{\frac{3}{2}}}\left(b_{i}^{2} d a_{i} \wedge d p-a_{i} b_{i} d a_{i} \wedge d q-a_{i} b_{i} d b_{i} \wedge d p+a_{i}^{2} d b_{i} \wedge d q\right) .
$$

From (11) we compute

$$
d \varphi_{i}=-\frac{1}{\left(a_{i}^{2}+b_{i}^{2}\right)^{\frac{3}{2}}}\left(a_{i} d a_{i}+b_{i} d b_{i}\right) .
$$

By exterior multiplication of the forms of (36) and (37) for $i=1,2$, we find

$$
d \varphi_{1} \wedge d \widetilde{G}_{1} \wedge d \varphi_{2} \wedge d \widetilde{G}_{2}=\frac{\sin \psi}{\varphi_{1}^{3} \varphi_{2}^{3}} d a_{1} \wedge d b_{1} \wedge d a_{2} \wedge d b_{2} \wedge d p \wedge d q
$$

and comparing with (31), we obtain

$$
d\left(G_{1}, G_{2}\right)=\varphi_{1}^{3} \varphi_{2}^{3} d \varphi_{1} \wedge d \widetilde{G}_{1} \wedge d \varphi_{2} \wedge d \widetilde{G}_{2} \wedge d r .
$$

Thus we are ready to state the following

THEOREM 4. The density for the pairs $\left(G_{1}, G_{2}\right)$ of intersecting nonisotropic straight lines of type $\alpha$, determined by (29), satisfies the relations (31), (33), (35) and (38).

3.2. Density of pairs of intersecting nonisotropic straight lines of type $\beta$. Let $\left(G_{1}, G_{2}\right)$ be a pair of intersecting nonisotropic straight lines of type $\beta$. Without loss of generality, we can assume that $G_{1}$ and $G_{2}$ have equations of the form

$$
\begin{aligned}
& G_{1}: \quad x=a_{1} z+p-a_{1} r, \quad y=b_{1} z+q-b_{1} r, \quad a_{1} \neq 0, \\
& G_{2}: \quad x=a_{2} z+p-a_{2} r, \quad y=\frac{a_{2}}{a_{1}} b_{1} z+q-\frac{a_{2}}{a_{1}} b_{1} r, \quad a_{2} \neq 0 .
\end{aligned}
$$

Then the corresponding associated group $\bar{B}_{6}^{(1)}$ has the infinitesimal operators

$$
\begin{aligned}
& Z_{1}=\frac{\partial}{\partial p}, \quad Z_{2}=\frac{\partial}{\partial q}, \quad Z_{3}=\frac{\partial}{\partial r}, \quad Z_{4}=b_{1} \frac{\partial}{\partial a_{1}}-a_{1} \frac{\partial}{\partial b_{1}}+\frac{a_{2}}{a_{1}} b_{1} \frac{\partial}{\partial a_{2}}+q \frac{\partial}{\partial p}-p \frac{\partial}{\partial q} \\
& Z_{5}=a_{1}^{2} \frac{\partial}{\partial a_{1}}+a_{1} b_{1} \frac{\partial}{\partial b_{1}}+a_{2}^{2} \frac{\partial}{\partial a_{2}}-p \frac{\partial}{\partial r}, \quad Z_{6}=a_{1} b_{1} \frac{\partial}{\partial a_{1}}+b_{1}^{2} \frac{\partial}{\partial b_{1}}+\frac{a_{2}^{2}}{a_{1}} b_{1} \frac{\partial}{\partial a_{2}}-q \frac{\partial}{\partial r}
\end{aligned}
$$

and it is intransitive. Consequently the set of pairs of intersecting straight lines of type $\beta$ is not measurable under $B_{6}^{(1)}$. 
The system $Z_{i}(f)=0, i=1, \ldots, 6$, has an independent integral

$$
f=\frac{a_{1}-a_{2}}{a_{2} \sqrt{a_{1}^{2}+b_{1}^{2}}}
$$

and it is an absolute invariant of $B_{6}^{(1)}$. We define the invariant density of the pairs $\left(G_{1}, G_{2}\right)\left(a_{1}, b_{1}, a_{2}, p, q, r\right)$ of type $\beta$ by the equality

$$
d\left(G_{1}, G_{2}\right)=\left|\frac{a_{1}-a_{2}}{a_{2} \sqrt{a_{1}^{2}+b_{1}^{2}}}\right| d a_{1} \wedge d b_{1} \wedge d a_{2} \wedge d p \wedge d q \wedge d r .
$$

In view of (20), (40) yields

$$
d\left(G_{1}, G_{2}\right)=|s| d a_{1} \wedge d b_{1} \wedge d a_{2} \wedge d p \wedge d q \wedge d r .
$$

In this case, we find

$$
d a_{1} \wedge d b_{1} \wedge d p \wedge d q \wedge d r=\frac{1}{r^{2}} d \bar{P}_{1} \wedge d P,
$$

where $\bar{P}_{1}=G_{1} \cap O x y, P=G_{1} \cap G_{2}$. But

$$
d s=\frac{a_{1} a_{2}+b_{1}^{2}}{a_{2}\left(a_{1}^{2}+b_{1}^{2}\right)^{\frac{3}{2}}} d a_{1}-\frac{\left(a_{1}-a_{2}\right) b_{1}}{a_{2}\left(a_{1}^{2}+b_{1}^{2}\right)^{\frac{3}{2}}} d b_{1}-\frac{a_{1}}{a_{2}^{2}\left(a_{1}^{2}+b_{1}^{2}\right)^{\frac{1}{2}}} d a_{2}
$$

and from (41), (42) and (43) we deduce

$$
d\left(G_{1}, G_{2}\right)=\left|\frac{a_{2}\left(a_{1}-a_{2}\right)}{a_{1} r^{2}}\right| d s \wedge d \bar{P}_{1} \wedge d P .
$$

We note that the straight lines $G_{1}$ and $G_{2}$ lie in the isotropic plane $\iota: b_{1} x-a_{1} y+$ $a_{1} q-b_{1} p=0$ and hence their orthogonal projections $\widetilde{G}_{1}$ and $\widetilde{G}_{2}$ coincide on $O x y$, i.e. $\widetilde{G}_{1} \equiv \widetilde{G}_{2} \equiv \widetilde{G}$. Then $\left(12^{\prime}\right),(37)$ and (43) imply

$$
d s \wedge d \varphi_{1} \wedge d \widetilde{G}=\frac{a_{1}}{\left(a_{1}^{2}+b_{1}^{2}\right)^{\frac{5}{2}}}\left(b_{1} d a_{1} \wedge d b_{1} \wedge d a_{2} \wedge d p-a_{1} d a_{1} \wedge d b_{1} \wedge d a_{2} \wedge d q\right) .
$$

From (40) and (45) we obtain

$$
d\left(G_{1}, G_{2}\right)=\left|\frac{s \varphi_{1}^{5}}{a_{1} b_{1}}\right| d s \wedge d \varphi_{1} \wedge d \widetilde{G} \wedge d q \wedge d r=\left|\frac{s \varphi_{1}^{5}}{a_{1}^{2}}\right| d s \wedge d \varphi_{1} \wedge d \widetilde{G} \wedge d p \wedge d r
$$

Therefore we have:

THEOREM 5. The density for the pairs $\left(G_{1}, G_{2}\right)$ of intersecting nonisotropic straight lines of type $\beta$, determined by (39), satisfies the relations (41), (44) and (46).

3.3. Density of pairs of intersecting nonisotropic and isotropic straight lines. Let $\left(G_{1}, G_{2}\right)$ be a pair of intersecting straight lines

$$
\begin{array}{ll}
G_{1}: & x=a_{1} z+p-a_{1} r, \quad y=b_{1} z+q-b_{1} r, \\
G_{2}: & x=p, \quad y=q,
\end{array}
$$


i.e. $G_{1}$ is nonisotropic and $G_{2}$ is isotropic. Now the corresponding associated group $\bar{B}_{6}^{(1)}$ has the infinitesimal operators

$$
\begin{aligned}
& U_{1}=\frac{\partial}{\partial p}, \quad U_{2}=\frac{\partial}{\partial q}, \quad U_{3}=\frac{\partial}{\partial r}, \quad U_{4}=b_{1} \frac{\partial}{\partial a_{1}}-a_{1} \frac{\partial}{\partial b_{1}}+q \frac{\partial}{\partial p}-p \frac{\partial}{\partial q}, \\
& U_{5}=a_{1}^{2} \frac{\partial}{\partial a_{1}}+a_{1} b_{1} \frac{\partial}{\partial b_{1}}-p \frac{\partial}{\partial r}, \quad U_{6}=a_{1} b_{1} \frac{\partial}{\partial a_{1}}+b_{1}^{2} \frac{\partial}{\partial b_{1}}-q \frac{\partial}{\partial r}
\end{aligned}
$$

and it is transitive. Then the integral invariant function $f=f\left(a_{1}, b_{1}, p, q, r\right)$ of the group $B_{6}^{(1)}$ satisfies the system of R. Deltheil [3; p. 28], [9; p. 11], namely

$U_{1}(f)=0, \quad U_{2}(f)=0, \quad U_{3}(f)=0, \quad U_{4}(f)=0, \quad U_{5}(f)+3 a_{1} f=0, \quad U_{6}(f)+3 b_{1} f=0$ and has the solution

$$
f=\frac{h}{\left(a_{1}+b_{1}\right)^{\frac{3}{2}}}
$$

where $h=$ const. Thus we have

THEOREM 6. The set of the pairs $\left(G_{1}, G_{2}\right)$ of intersecting straight lines (47) is measurable with respect to $B_{6}^{(1)}$ and the corresponding invariant density is

$$
d\left(G_{1}, G_{2}\right)=\frac{1}{\left(a_{1}^{2}+b_{1}^{2}\right)^{\frac{3}{2}}} d a_{1} \wedge d b_{1} \wedge d p \wedge d q \wedge d r .
$$

From (11) and (48) it follows immediately that

$$
d\left(G_{1}, G_{2}\right)=\varphi_{1}^{3} d a_{1} \wedge d b_{1} \wedge d p \wedge d q \wedge d r .
$$

On the other hand, by direct computation we obtain

$$
d a_{1} \wedge d b_{1} \wedge d p \wedge d q=\frac{1}{r^{2}} d \widetilde{P} \wedge d \bar{P},
$$

where $\widetilde{P}=G_{1} \cap O x y$ and $\bar{P}=G_{2} \cap O x y$. Applying (50) to (49) we get

$$
d\left(G_{1}, G_{2}\right)=\frac{\varphi_{1}^{3}}{r^{2}} d r \wedge d \widetilde{P} \wedge d \bar{P}
$$

Similarly, from (49) and

$$
d a_{1} \wedge d b_{1} \wedge d p \wedge d q \wedge d r=-\frac{1}{\varphi_{1} r^{3}} d \varphi_{1} \wedge d \widetilde{P} \wedge d \bar{P}
$$

we find

$$
d\left(G_{1}, G_{2}\right)=\left|\frac{\varphi_{1}^{2}}{r^{3}}\right| d \varphi_{1} \wedge d \widetilde{P} \wedge d \bar{P} .
$$

We establish the following result:

THEOREM 7. The density for the pairs $\left(G_{1}, G_{2}\right)$ of intersecting straight lines (47) satisfies the relations (49), (51) and (52).

\section{Measurability of a set of pairs of parallel straight lines}

4.1. Density of pairs of parallel nonisotropic straight lines of different isotropic planes. Let $\left(G_{1}, G_{2}\right)$ be a pair of parallel nonisotropic straight lines 


$$
\begin{aligned}
& G_{1}: \quad x=a z+p_{1}, \quad y=b z+q_{1}, \\
& G_{2}: \quad x=a z+p_{2}, \quad y=b z+q_{2}, \quad a^{2}+b^{2} \neq 0
\end{aligned}
$$

that lie in different isotropic planes, i.e.

$$
a\left(q_{2}-q_{1}\right)-b\left(p_{2}-p_{1}\right) \neq 0 .
$$

The corresponding associated group $\bar{B}_{6}^{(1)}$ has the infinitesimal operators

$$
\begin{aligned}
& Y_{1}=\frac{\partial}{\partial p_{1}}+\frac{\partial}{\partial p_{2}}, Y_{2}=\frac{\partial}{\partial q_{1}}+\frac{\partial}{\partial q_{2}}, Y_{3}=a Y_{1}+b Y_{2}, \\
& Y_{4}=-b \frac{\partial}{\partial a}+a \frac{\partial}{\partial b}-q_{1} \frac{\partial}{\partial p_{1}}+p_{1} \frac{\partial}{\partial q_{1}}-q_{2} \frac{\partial}{\partial p_{2}}+p_{2} \frac{\partial}{\partial q_{2}}, \\
& Y_{5}=a^{2} \frac{\partial}{\partial a}+a b \frac{\partial}{\partial b}+a p_{1} \frac{\partial}{\partial p_{1}}+b p_{1} \frac{\partial}{\partial q_{1}}+a p_{2} \frac{\partial}{\partial p_{2}}+b p_{2} \frac{\partial}{\partial q_{2}}, \\
& Y_{6}=a b \frac{\partial}{\partial a}+b^{2} \frac{\partial}{\partial b}+a q_{1} \frac{\partial}{\partial p_{1}}+b q_{1} \frac{\partial}{\partial q_{1}}+a q_{2} \frac{\partial}{\partial p_{2}}+b q_{2} \frac{\partial}{\partial q_{2}}
\end{aligned}
$$

and obviously it is intransitive. Hence the set of pairs of parallel nonisotropic straight lines (53), $\left(53^{\prime}\right)$ is not measurable with respect to $B_{6}^{(1)}$.

On the other hand, the value

$$
f=\frac{a\left(q_{2}-q_{1}\right)-b\left(p_{2}-p_{1}\right)}{\sqrt{a^{2}+b^{2}}}
$$

is an absolute invariant of $\bar{B}_{6}^{(1)}$ and we define the density for the pairs $\left(G_{1}, G_{2}\right)\left(a, b, p_{1}\right.$, $\left.q_{1}, p_{2}, q_{2}\right)$ by the equality

$$
d\left(G_{1}, G_{2}\right)=\left|\frac{a\left(q_{2}-q_{1}\right)-b\left(p_{2}-p_{1}\right)}{\sqrt{a^{2}+b^{2}}}\right| d a \wedge d b \wedge d p_{1} \wedge d q_{1} \wedge d p_{2} \wedge d q_{2} .
$$

REMARK 4 . The parallel straight lines on the coordinate plane $O x y$

$$
\widetilde{G}_{1}: \quad b x-a y+a q_{1}-b p_{1}=0, \quad z=0
$$

and

$$
\widetilde{G}_{2}: \quad b x-a y+a q_{2}-b p_{2}=0, \quad z=0
$$

are the orthogonal projections of the parallel straight lines $G_{1}$ and $G_{2}$, respectively. Then the Euclidean distance between $\widetilde{G}_{1}$ and $\widetilde{G}_{2}$ is

$$
\delta\left(\widetilde{G}_{1}, \widetilde{G}_{2}\right)=\left|\frac{a\left(q_{2}-q_{1}\right)-b\left(p_{2}-p_{1}\right)}{\sqrt{a^{2}+b^{2}}}\right| .
$$

Putting (55) into (54) we get

$$
d\left(G_{1}, G_{2}\right)=\delta\left(\widetilde{G}_{1}, \widetilde{G}_{2}\right) d a \wedge d b \wedge d p_{1} \wedge d q_{1} \wedge d p_{2} \wedge d q_{2} .
$$

A computation leads to

$$
d \delta \wedge d \varphi \wedge d \bar{P}_{1} \wedge d \bar{P}_{2}=-\frac{a\left(p_{2}-p_{1}\right)+b\left(q_{2}-q_{1}\right)}{\left(a^{2}+b^{2}\right)^{2}} d a \wedge d b \wedge d p_{1} \wedge d q_{1} \wedge d p_{2} \wedge d q_{2},
$$

where $\delta=\delta\left(\widetilde{G}_{1}, \widetilde{G}_{2}\right), \varphi=\angle\left(G_{1}, O x y\right)=\angle\left(G_{2}, O x y\right), \bar{P}_{1}=G_{1} \cap O x y, \bar{P}_{2}=G_{2} \cap O x y$. 
In view of (57), (56) yields

$$
d\left(G_{1}, G_{2}\right)=\left|\frac{\delta}{\varphi^{4}\left[a\left(p_{2}-p_{1}\right)+b\left(q_{2}-q_{1}\right)\right]}\right| d \delta \wedge d \varphi \wedge d \bar{P}_{1} \wedge d \bar{P}_{2} .
$$

THEOREM 8. The density for the pairs $\left(G_{1}, G_{2}\right)$ of parallel nonisotropic straight lines (53), (53') satisfies the relations (56) and (58).

4.2. Density of pairs of parallel nonisotropic straight lines of coinciding isotropic planes. Let $\left(G_{1}, G_{2}\right)$ be a pair of parallel nonisotropic straight lines that lie in an isotropic plane. Assume that $G_{1}$ and $G_{2}$ have the equations

$$
\begin{array}{ll}
G_{1}: & x=a z+p_{1}, \quad y=b z+q_{1}, \quad a \neq 0, \\
G_{2}: & x=a z+p_{2}, \quad y=b z+q_{1}+\frac{b}{a}\left(p_{2}-p_{1}\right), \quad b \neq 0 .
\end{array}
$$

Then the corresponding associated group has the infinitesimal operators

$$
\begin{aligned}
& Z_{1}=\frac{\partial}{\partial p_{1}}+\frac{\partial}{\partial p_{2}}, \quad Z_{2}=\frac{\partial}{\partial q_{1}}, \quad Z_{3}=a Z_{1}+b Z_{2}, \\
& Z_{4}=-b \frac{\partial}{\partial a}+a \frac{\partial}{\partial b}-q_{1} \frac{\partial}{\partial p_{1}}+p_{1} \frac{\partial}{\partial q_{1}}-\left[q_{1}+\frac{b}{a}\left(p_{2}-p_{1}\right)\right] \frac{\partial}{\partial p_{2}}, \\
& Z_{5}=a^{2} \frac{\partial}{\partial a}+a b \frac{\partial}{\partial b}+a p_{1} \frac{\partial}{\partial p_{1}}+b p_{1} \frac{\partial}{\partial q_{1}}+a p_{2} \frac{\partial}{\partial p_{2}}, \\
& Z_{6}=a b \frac{\partial}{\partial a}+b^{2} \frac{\partial}{\partial b}+a q_{1} \frac{\partial}{\partial p_{1}}+b q_{1} \frac{\partial}{\partial q_{1}}+a\left[q_{1}+\frac{b}{a}\left(p_{2}-p_{1}\right)\right] \frac{\partial}{\partial p_{2}}
\end{aligned}
$$

and it is intransitive. Therefore the set of pairs of parallel nonisotropic straight lines (59) is not measurable with respect to $B_{6}^{(1)}$. The system $Z_{i}(f)=0, i=1, \ldots 6$, has the solution

$$
f=\frac{p_{2}-p_{1}}{a}
$$

and it is an absolute invariant of $\bar{B}_{6}^{(1)}$. Then we define the density for the pairs $\left(G_{1}, G_{2}\right)$ $\left(a, b, p_{1}, q_{1}, p_{2}\right)$ by the equality

$$
d\left(G_{1}, G_{2}\right)=\left|\frac{p_{2}-p_{1}}{a}\right| d a \wedge d b \wedge d p_{1} \wedge d q_{1} \wedge d p_{2} .
$$

REMARK 5. The isotropic plane $\iota: a x+b y=0$ intersects the straight lines $G_{1}$ and $G_{2}$ at the points

$$
P_{1}\left(-\frac{b\left(a q_{1}-b p_{1}\right)}{a^{2}+b^{2}}, \frac{a\left(a q_{1}-b p_{1}\right)}{a^{2}+b^{2}},-\frac{a p_{1}+b q_{1}}{a^{2}+b^{2}}\right)
$$

and

$$
P_{2}\left(-\frac{b\left(a q_{1}-b p_{1}\right)}{a^{2}+b^{2}}, \frac{a\left(a q_{1}-b p_{1}\right)}{a^{2}+b^{2}},-\frac{\left(a^{2}+b^{2}\right) p_{2}+b\left(a q_{1}-b p_{1}\right)}{a\left(a^{2}+b^{2}\right)}\right),
$$

respectively. Obviously $P_{1}$ and $P_{2}$ are parallel points and then

$$
s\left(P_{1}, P_{2}\right)=\frac{p_{1}-p_{2}}{a},
$$

i.e. (61) is the oriented $s$-distance from $G_{1}$ to $G_{2}$. Further we shall denote $s\left(G_{1}, G_{2}\right)=$ $s\left(P_{1}, P_{2}\right)$ by $s$. 
Hence by (60) and (61) we have

$$
d\left(G_{1}, G_{2}\right)=|s| d a \wedge d b \wedge d p_{1} \wedge d q_{1} \wedge d p_{2} .
$$

We compute

$$
d a \wedge d b \wedge d p_{1} \wedge d q_{1} \wedge d p_{2}=\frac{a}{s^{2}} d s \wedge d \bar{P}_{1} \wedge d \bar{P}_{2}
$$

and

$$
d a \wedge d b \wedge d p_{1} \wedge d q_{1} \wedge d p_{2}=-\frac{a}{s \varphi} d \varphi \wedge d \bar{P}_{1} \wedge d \bar{P}_{2}
$$

where $\bar{P}_{1}=G_{1} \cap O x y, \bar{P}_{2}=G_{2} \cap O x y, \varphi=\angle\left(G_{1}, O x y\right)=\angle\left(G_{2}, O x y\right)$. Substituting (63) and (64) into (62), we find

$$
d\left(G_{1}, G_{2}\right)=\left|\frac{a}{s}\right| d s \wedge d \bar{P}_{1} \wedge d \bar{P}_{2}
$$

and

$$
d\left(G_{1}, G_{2}\right)=\left|\frac{a}{\varphi}\right| d \varphi \wedge d \bar{P}_{1} \wedge d \bar{P}_{2},
$$

respectively. Thus we have the following

TheOREM 9. The density for the pairs $\left(G_{1}, G_{2}\right)$ of parallel nonisotropic straight lines (59) satisfies the relations (62), (65) and (66).

4.3. Density of pairs of isotropic straight lines. Let $\left(G_{1}, G_{2}\right)$ be a pair of isotropic straight lines and

$$
G_{1}: \quad x=p_{1}, \quad y=q_{1}, \quad G_{2}: \quad x=p_{2}, \quad y=q_{2},
$$

where $\left(p_{2}-p_{1}\right)^{2}+\left(q_{2}-q_{1}\right)^{2} \neq 0$. The corresponding associated group $\bar{B}_{6}^{(1)}$ has the infinitesimal operators

$$
\begin{aligned}
& U_{1}=\frac{\partial}{\partial p_{1}}+\frac{\partial}{\partial p_{2}}, \quad U_{2}=\frac{\partial}{\partial q_{1}}+\frac{\partial}{\partial q_{2}}, \quad U_{3}=0 \\
& U_{4}=-q_{1} \frac{\partial}{\partial p_{1}}+p_{1} \frac{\partial}{\partial q_{1}}+q_{2} \frac{\partial}{\partial p_{2}}-p_{2} \frac{\partial}{\partial q_{2}}, \quad U_{5}=0, \quad U_{6}=0
\end{aligned}
$$

and it is intransitive. It follows that the set of pairs of isotropic straight lines is not measurable with respect to $B_{6}^{(1)}$. But

$$
f=\left(p_{2}-p_{1}\right)^{2}+\left(q_{2}-q_{1}\right)^{2}
$$

is an absolute invariant of $\bar{B}_{6}^{(1)}$ and we can define the density for the pairs $\left(G_{1}, G_{2}\right)$ $\left(p_{1}, q_{1}, p_{2}, q_{2}\right)$ by the equality

$$
d\left(G_{1}, G_{2}\right)=\sqrt{\left(p_{2}-p_{1}\right)^{2}+\left(q_{2}-q_{1}\right)^{2}} d p_{1} \wedge d q_{1} \wedge d p_{2} \wedge d q_{2} .
$$

REMARK 6. We note that $[7 ;$ p. 46]

$$
l^{\star}=\sqrt{\left(p_{2}-p_{1}\right)^{2}+\left(q_{2}-q_{1}\right)^{2}}
$$

is the distance between $G_{1}$ and $G_{2}$. 
Inserting (15) and (69) into (68) we obtain

$$
d\left(G_{1}, G_{2}\right)=l^{\star} d \bar{P}_{1} \wedge d \bar{P}_{2} .
$$

On the other hand, we have

and therefore

$$
\begin{aligned}
d \bar{P}_{1} \wedge d \bar{P}_{2} & =-\frac{1}{p_{2}-p_{1}} d l^{\star} \wedge d q_{1} \wedge d \bar{P}_{2}=-\frac{1}{q_{2}-q_{1}} d p_{1} \wedge d l^{\star} \wedge d \bar{P}_{2} \\
& =\frac{1}{p_{2}-p_{1}} d \bar{P}_{1} \wedge d l^{\star} \wedge d q_{2}=\frac{1}{q_{2}-q_{1}} d \bar{P}_{1} \wedge d p_{2} \wedge d l^{\star}
\end{aligned}
$$

$$
\begin{aligned}
d\left(G_{1}, G_{2}\right) & =\frac{l^{\star}}{\left|p_{2}-p_{1}\right|} d l^{\star} \wedge d q_{1} \wedge d \bar{P}_{2}=\frac{l^{\star}}{\left|q_{2}-q_{1}\right|} d p_{1} \wedge d l^{\star} \wedge d \bar{P}_{2} \\
& =\frac{l^{\star}}{\left|p_{2}-p_{1}\right|} d \bar{P}_{1} \wedge d l^{\star} \wedge d q_{2}=\frac{l^{\star}}{\left|q_{2}-q_{1}\right|} d \bar{P}_{1} \wedge d p_{2} \wedge d l^{\star} .
\end{aligned}
$$

Thus we have the following

THEOREM 10. The density for the pairs $\left(G_{1}, G_{2}\right)$ of isotropic straight lines (67) satisfies the relations (70) and (71).

\section{References}

[1] W. Blaschke, Vorlesungen über Integralgeometrie, Third edition, Deutsch. Verlag Wiss., Berlin, 1955.

[2] A. Borisov and M. Spirova, Some integral geometric results in the simply isotropic space, J. Geom. 77 (2003), 8-21.

[3] R. Deltheil, Sur la théorie des probabilités géométriques, Ann. Fac. Sc. Univ. Toulouse 3 (1919), 1-65.

[4] G. I. Drinfel'd, On the measure of the Lie groups, Zap. Mat. Otdel. Fiz. Mat. Fak. Kharkov. Mat. Obsc. 21 (1949), 47-57 (in Russian).

[5] G. I. Drinfel'd and A. V. Lucenko, On the measure of sets of geometric elements, Vest. Kharkov. Univ. 31 (1964), no. 3, 34-41 (in Russian).

[6] A. V. Lucenko, On the measure of sets of geometric elements and their subset, Ukrain. Geom. Sb. 1 (1965), 39-57 (in Russian).

[7] H. Sachs, Isotrope Geometrie des Raumes, Vieweg, Braunschweig/Wiesbaden, 1990.

[8] L. A. Santaló, Integral Geometry and Geometric Probability, Addison-Wesley, 1976.

[9] M. I. Stoka, Geometrie Integrală, Ed. Acad. RSR, Bucuresti, 1967.

[10] M. I. Stoka, Géométrie Intégrale, Mém. Sci. Math. 165, Gauthier-Vilars, Paris, 1968. 